\title{
How to assess the severity of bronchiectasis
}

\author{
Aarash D. Saleh and John R. Hurst \\ Affiliations: UCL Respiratory Medicine, University College London, London, UK. \\ Correspondence: J.R. Hurst, University College London, London, London, UK. E-mail: j.hurstaucl.ac.uk
}

@ERSpublications

When assessing the 'severity' of bronchiectasis, consider both imaging and clinical variables http:// ow.ly/tGRLS

Wait long enough for a bus and two arrive at once. As with buses, so with bronchiectasis scoring systems. In this issue of the European Respiratory Journal MARTINEZ-GARCIA et al. [1] report the development of a "FACED" bronchiectasis prognostic score. This follows the recent publication by CHALMERS et al. [2] of the "Bronchiectasis Severity Index" (BSI). Bronchiectasis is a neglected disease and research in the area is to be welcomed, especially when it enables us to manage our patients more appropriately. But, like any good research, these studies raise as many questions as answers: most importantly, what do we even mean when we talk about bronchiectasis "severity"?

Current definitions of bronchiectasis have two components. The landmark British Thoracic Society guidance emphasises the concept of "clinically significant" bronchiectasis [3]; the presence of "symptoms of persistent or recurrent bronchial sepsis", in addition to the permanent airway structural damage that represents the accepted pathology definition. Tools to grade the degree and extent, or "severity" of the radiological component already exist; e.g. scoring systems described by Reiff et al. [4] and Bhalla et al. [5]. These are not without problems. Importantly, patients can have similar severity scores arising from structurally more abnormal but localised disease, or more widespread but less prominent airway-wall thickening and dilatation on computed tomography (CT) (fig. 1). These processes are likely to represent very different aetiologies and natural history. Moreover, the amount and extent of pathology does not represent the rate of airway damage: a concept that might best be called disease activity. To use a second transport analogy, if pathological severity represents the distance travelled on a journey from lung health to lung disease, disease activity represents the speed of that journey. Severe disease need not be active, and active disease need not (yet) be severe.

Therefore, it is clear that there are two key problems in defining the severity of pathology in bronchiectasis. The next consideration is the complex relationship between the severity of radiological disease and that of the second component, the clinical syndrome. We will all recognise patients with apparently trivial anatomical distortion who experience a great burden of symptoms and/or exacerbations, and conversely there are patients with greater degrees of airway destruction who appear resistant.

A better assessment of bronchiectasis "severity" might, therefore, combine assessments of pathological state with clinical syndrome. Both FACED and BSI do just this, but to what end? What is the point of a bronchiectasis score? A useful severity score might allow us to predict important outcomes such as exacerbations, hospitalisations or death, or to identify groups of patients that may respond to a particular therapy. Better yet, a score may respond to changes in disease status with changes in therapy. This raises the exciting possibility that the score itself may find use as an outcome measure in the clinic or clinical trials. At a more basic level, a widely accepted score would at the very least allow comparison of patients across studies.

Received: Dec 272013 | Accepted after revision: Jan 132014

Conflict of interest: Disclosures can be found alongside the online version of this article at www.erj.ersjournals.com

Copyright (CERS 2014 

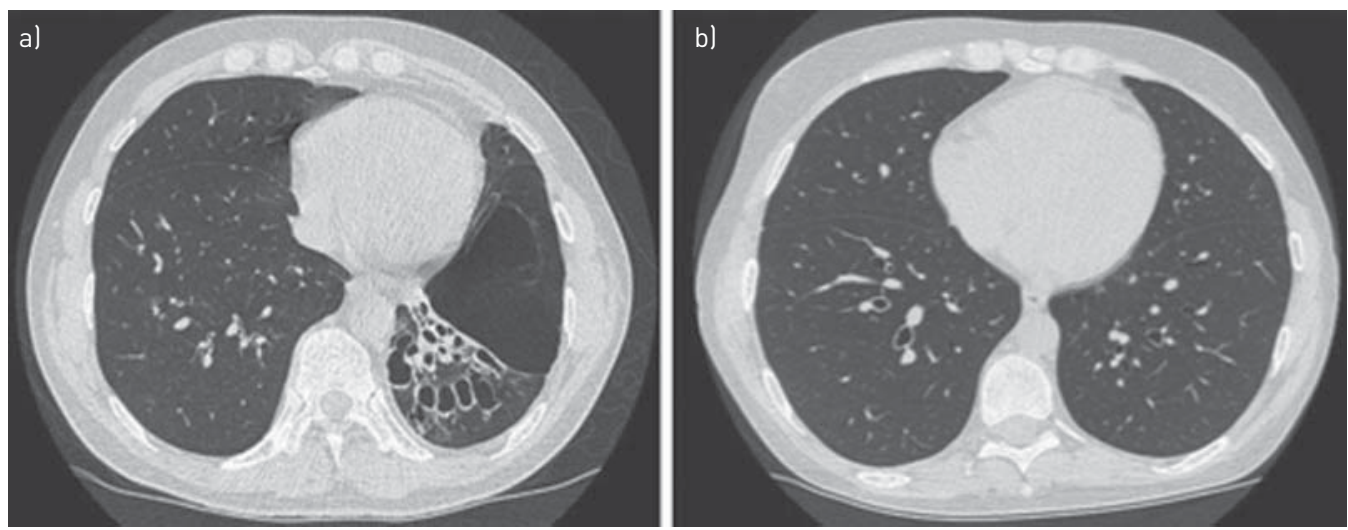

FIGURE 1 A similar total imaging-based "severity" score may result from: a) a localised destructive process or b) a more widespread, but less prominent disease, when present throughout the lungs.

FACED was designed as a prognostic score [1]. The strengths of the work include the use of separate development and validation datasets (though from the same cohort), and the inclusion of variables that are relatively simple to collect in clinical practice: $\mathrm{F}$ for forced expiratory volume in $1 \mathrm{~s}$ (FEV1); A for Age; C for Pseudomonas colonisation; E for extent of bronchiectasis (counting lobar involvement on a CT scan); and D for dyspnoea, using the Medical Research Council dyspnoea score. All the variables were dichotomised and scored 0 versus 1 or 2, depending on the strength of association with mortality in the initial analysis. The total score predicted the 5-year all-cause mortality in the validation data with an area under a receiveroperating characteristic curve (AUC) of 0.83 . To provide clinical context, this equates to a 5 -year mortality in mild, moderate and severe disease (defined as a score of $0-2,3-4$ and $5-7$, respectively) of $4 \%, 25 \%$ and $56 \%$, respectively. Factors predictive of mortality in bronchiectasis have been examined previously [6], and associations between lung function, Pseudomonas carriage and symptoms (or health status) described. The value of FACED is the use of these data to construct a clinically meaningful score. Note that the score was calculated at the time of initial diagnosis (and is not, therefore, designed for the follow-up of existing patients, which is an important limitation) and we do not know if a change in score reflects a change in prognosis.

The BSI combines age, body mass index, FEV1, previous hospitalisation, exacerbation frequency, colonisation status, and radiological appearances. The score was designed to predict future exacerbations and hospitalisations, health status and death over 4 years. As with FACED, the study was carefully conducted and the BSI was validated in multiple international cohorts. Patients with non-tuberculous Mycobacteria were excluded. Importantly, data are provided that examine how the score performed in an annual prediction of events, a more relevant clinical scenario, and the team have developed a useful online calculator [7]. The mortality AUC in the validation cohorts was similar to that achieved with the simpler FACED score.

Reassuringly, coming from different groups and cohorts, the two scores include similar variables. There may be some potentially relevant omissions, notably the presence of comorbidities, which we know to be important in the prognosis of other chronic respiratory diseases. Finally, it seems unlikely that either score can readily be used as an outcome measure, given the irreversibility of anatomical destruction, airflow obstruction and age. What now? FACED and BSI predict long-term mortality in bronchiectasis similarly well; FACED is simpler whilst BSI, though more complex, also informs on annual risk and the additional outcome of hospitalisation. However, what we do not yet know is whether use of these scores to identify high-risk groups can improve outcomes. And this is the challenge going forward: how can we reduce symptoms and exacerbations, preserve lung function and functional status, and prevent premature mortality in our patients? We can't do this with FACED or BSI alone, but use of these tools may be the first step to a brighter future for our patients with this important and neglected condition.

\section{References}

1 Martínez-García MÁ, de Gracia J, Vendrell Relat M, et al. Multidimensional approach to non-cystic fibrosis bronchiectasis: the FACED score. Eur Respir J 2014: 43; 1357-1367.

2 Chalmers JD, Goeminne P, Aliberti S, et al. The Bronchiectasis Severity Index: an international derivation and validation study. Am J Respir Crit Care Med 2014 [In press DOI. 10.1164/rccm.201309-1575OC].

Pasteur MC, Bilton D, Hill AT, et al. British Thoracic Society guideline for non-CF bronchiectasis. Thorax 2010; 65: Suppl. 1, i1-i58. 
4 Reiff DB, Wells AU, Carr DH, et al. CT findings in bronchiectasis: limited value in distinguishing between idiopathic and specific types. Am J Radiol 1995; 165: 261-267.

5 Bhalla M, Turcios N, Aponte V, et al. Cystic fibrosis: scoring system with thin-section CT. Radiology 1991; 179: 783-788.

6 Loebinger MR, Wells UA, Hansell DM, et al. Mortality in bronchiectasis: a long-term study assessing the factors influencing survival. Eur Respir J 2009; 34: 843-849.

7 Chalmers JD, Goeminne P, Aliberti S, et al. Bronchiectasis Severity Index: predicting mortality and exacerbation rates in non-CF bronchiectasis. www.bronchiectasisseverity.com/ Date last accessed December 23, 2013. 